\title{
A New Method to Protect Video using the Watermark Embedded by SWT Transform and Genetic Algorithm
}

\author{
Mahmoud Rahhal \\ Aleepo, Syria \\ Computer Engineering Department, \\ College of Electrical and Electronic Engineering, \\ University of Aleppo
}

\begin{abstract}
This research, design a watermarking system for embedding and extracting the watermark in video files. This research presented a new method that is efficient and powerful to embed and extract the watermark, which relied on the conversion of SWT (Stationary Wavelet Transform) on each data frame of the video file that required for by, then this method has been improved by using a genetic algorithm for embedding the watermark. Watermark was efficient and robust against various attacks which was found by measuring the efficiency of the method by calculating the values of the PSNR (Peak Signal-toNoise Ratio) and the NC (Normalized correlation Coefficient).
\end{abstract}

\section{Keywords}

Watermarking, Wavelet Domain, Stationary Wavelet Transform, embed ,extract, Cover, Genetic , PSNR , NC, Video, Frames.

\section{INTRODUCTION}

We live on a development world in sciences and technologies, the digital information exchanged rapidly and easily by the Internet or any computer network with little cost so easy copy, rigging or data transform in incorrect methods become simple so was must depend on watermark technique which is one of the branches of data hide and of the solution for recognition illegal copies and discover the rigging so you can hide large amount of data invisibly in the cover (the holder signal) and to be the watermark useful should be strongest against the operation discovery and probability attacks.

The techniques of watermarking classification by the domain which is use it in (spatial, frequencies),spatial domain depend on posturing pixels in the holder cover and choice the embedding points randomly whereas the frequencies domain more stronger than the spatial domain because use the frequencies values (more transparence and effect ) [1] which produced from use one of the method which depend on the frequency domain(SWT,DWT,SVD,DCT,....).The watermark technique is developed firstly for images and videos data and lately for the sound.

There are many of vocabularies in watermark technique should illustration it: the cover (the holder signal) which must embedding the information in it maybe was image, video or sound, watermark digital information add to the cover for extract later for discover the rigging the watermark maybe was image, number or paragraph [1],embedding the method of embed digital information (the watermark) in other signal (image ,video ,sound,........), extracting the method of extract information from watermark to use it in proof owner rights and discover the rigging if was found. the theories of watermark Similar in the methods of extract and embed. called the hide method which doesn't need any precast information between sender and receiver and doesn't need for key in the hide and extract method for data the simple hide [2].

Input of the system consist of the cover (the proposed video) and the watermark whereas output it consist of the cover after embedding the watermark into [1], in almost times we use the keys through the operation of embedding watermarking for make the watermark difficult for burrowing and more resist for delete, wiliness and print-scan operations whereas the extraction operation completed by entire the data of cover after embedding the watermark in the cover and the key which use before in embedding operation.

This paper is organized in the following sections, section 2 shows the Importance of watermarking technique, and section 3 shows Classification of digital watermarking, and section 4 shows the proposed method SWT with embedding algorithm, section 5 shows the GA and use it in embedding operation, finally section 6 compares the results and discussions this approach values PSNR\&\&NC.

\section{IMPORTANCE OF WATERMARKING TECHINQUE}

This research goals to development the embedding and extracting the watermarking method in video files by using SWT transform on all data in every frame of video frames which we need it for embedding process after that we combine this method with artificial intelligence genetic algorithm (GA) which discover the best situations for embedding and calculate the ability of this proposed algorithm in resist the various attacks (copy column and rows randomly, Gaussian filter, noise salt\&\&pepper and jpeg compression) through calculate the fitness function which depend on the quality of cover (PSNR) and accuracy of extract the watermark (NC).

The importance of this proposed method through determine the best places which include embedding in it and which gave high robustness against the various attacks causes discover the rigging and protect the right owners. in the watermarking system there are many standards we should consider it like (Robustness, Tamper Resistance, Fidelity) [1].

\subsection{Classification of digital watermarking}

There are many algorithms which are being used to hide the secret in-formation. These algorithms can be categorized into tow domains called: spatial domain and frequency domain.

Spatial domain watermarking slightly modifies the pixels of one or two randomly selected subsets of an image. On the other side, in frequency domain techniques the image is first transformed to the frequency domain by the use of any transformation methods such as Fourier transform, discrete cosine transform (DCT) or discrete wavelet transform (DWT). 
Now the information is added to the values of its transform coefficients. After applying the inverse transform, the marked coefficients form the embedded image. [3]

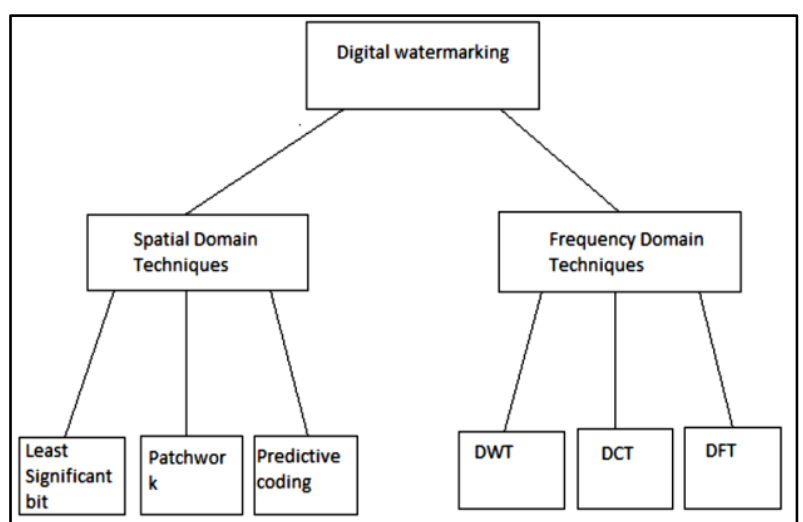

Fig 1 - Classification of Digital watermarking

\section{Related works}

Represents the researcher ASLANTS [2] method for embedding the watermark in digital images depend on SVD with GA through the scale operand $\mathrm{K}$ where he applied SVD on image I then update matrix values $\mathrm{S}$ with the watermark W so obtained on the updated unique values matrix Sm. Represent the researches in [4] new method for embedding the watermark in digital images by SWT with GA and depend on PSO where gave this proposed method good robustness and transparence results. Represent the researches in [5] new method with SWT transform after divide the image into four blocks after that apply one wavelet levels and three wavelet levels where the values of PSNR was good. compare the researches in [6] between the GA and other famous search algorithms (FA, CSO, PSO, CS) where was the GA the best of intelligence algorithm from speed and arrive to the best solution. Represent the researchers Shelke\&\& Chatur in [7] new method for embedding the watermark in video files by combine many of transforms like (DWT,PCA,SWT,SVD) transform with GA where they use the operand PSNR as the fitness function and shows good results against many of attacks especially against compression attack(jpeg) on the other hand, this method doesn't work only with the small videos because complex architecture result combine many transforms of DWT and deals with one form avi and doesn't show any resist against the many of attacks.

\section{STATIONARY WAVELET TRANSFORM (SWT)}

This transform is a wavelet transform which designed for overcome on weakness in resist the displacing in DWT algorithm through delete two levels of up and down samples during work of the DWT algorithm by using the operator $(2)^{j-1}$ in the third level of algorithm [6].

Swt is transform with residual that we take in the output number of samples equal to the input samples signals.

The goal of Swt transform to divide every frame of frames video files into four beams by use high-low filters in figure 2 :

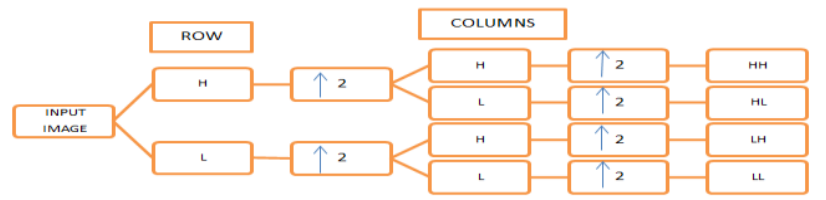

Fig 2: the SWT algorithm

Use the equations (1), (2) for SWT embedding and extracting:

$$
\begin{aligned}
& S W C=\operatorname{swt} 2 \text { (X`N،'wname') } \\
& X=\text { iswt2(SWC،N،'wname') }
\end{aligned}
$$

$X$ : the frames which we should applying SWT into.

$\mathrm{N}$ : the number of levels should be positive from complications of $(2)^{n}$

Wname: kind of waves that we should apply that there are many waves we can use it.

Swc: the output (horizontal, vertical, diameter and approximations).

According to the before characteristics we use the Swt transform in embedding the watermark in this paper. The operation of embedding and extracting materialized by generate the random keys (choose the best local for embedding operation) in the wavelet transform and update this values for embedding the watermark in it [8].

\subsection{We summarized the automatism work of the proposed SWT embedding approach as shown in figure (3)}

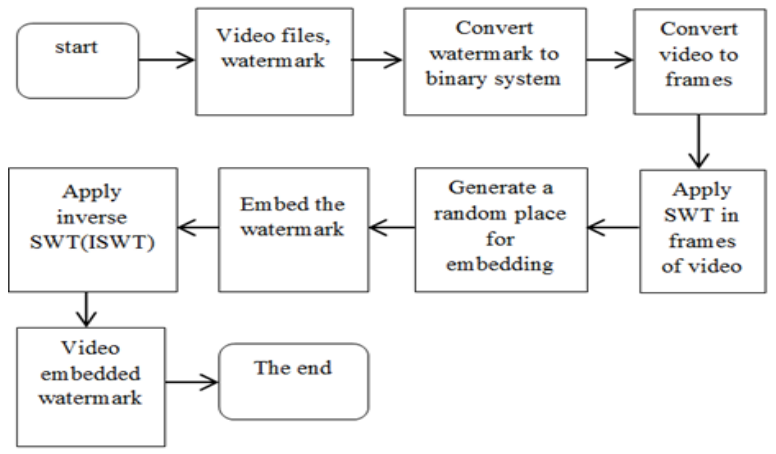

Fig 3: the SWT embedding proposed approach

1. read the video VI and the watermark with dimensions $n * m$.

2. convert the watermark to serial of bits $\mathrm{Wi}$ where $\mathrm{Wi}$ 0 or 1 .

3. convert every frame $\mathrm{f}$ of video from RGB to Ycber and choose the matrix $\mathrm{Y}$ for embedding in it (this form Ycbcr convert the colorful frames to three layers $\mathrm{Y}$ represent the intensity brightening for image and $\mathrm{Cb}$ represent the blue color and $\mathrm{Cr}$ represent the red color which showed the last studies that Ycbcr system more effective and protect against many attacks which may be exposed the image otherwise, the Ycbcr more transparency than RGB moreover, programming the Ycbcr easier than RGB because independence the layer of brightening from the layers of the color for that we convert to Ycbcr form but we use the $\mathrm{Y}$ layer because the human eye less sensitive for changes in the Y layer[9]).

4. Apply the SWT transform in every frame of video frames depending on the equation (2):

[swtimages‘swp]= swt2 (double (frame(i))،1،'Haar') Where: 
SWP: the matrix of approximate operands.

Swtimages : the matrix of details operands (horizontal, vertical, diameter).

Frame: the frames of video which embedded in it.

i: the number of frames (1. number of frames video).

Haar: the wave kind which used it.

5. Generate keys for specific the necessary places for embedding the watermark in the origin cover depending on equation (4) which give right places values within the size the chosen video, we depend on the dimensions of the watermark for calculate the length of the key depend on equation (3):

Length $($ key $)=n * m$.

keys $=$ round $(\quad$ (mimage*nimage $) / 4)+1) \quad *$ $(\operatorname{rand}(\operatorname{size}(1 \cdot 1)$ ،length $(\log 0))+1) \ldots . .(4)$

where:

mimage: the number of rows of frames.

nimage: the number of column of frames.

6. Embedding the watermark in the pixels which we specified in the previous step within the pixels of frame depend on equation (5):

swtimages $(\operatorname{keys}(\mathrm{i}))=$ swtimages $(\operatorname{keys}(\mathrm{i}))+$

$\alpha * \log$ o(i)......

where:

logo: the watermark which use it.

$\alpha$ : the power embedding operand.

Keys: the random keys which we generated in step 5 .

Swtimages: the matrixes (horizontal, vertical, diameter and approximations) for handle frame.

$\mathrm{i}$ : the counter of loop (1... length (key)).

7. Apply the inverse SWT (ISWT) on every frame of video frames and obtainment the frames which have the watermark.

8. Convert the video frames $\mathrm{f}^{\prime}$ from Ycbcr to RGB.

9. Rebuild the video frames VI' which embedded the watermark and show the video VI'.

10. Calculate the PSNR between the frames video VI which embedded the watermark and frames video VI.

11. Apply some attacks on video VI like (jpeg attack or salt\&\&pepper and others) for calculate the NC to specific quality of the extract watermark [10].

\subsection{We summarized the automatism work of the proposed SWT extracting approach as shown in figure (4)}

This operation starts by input the video VI' which contain the watermark to the proposed system in extract level where divide the frames of video VI' for extract the watermark from every frame of video frame that obvious in fig 4.

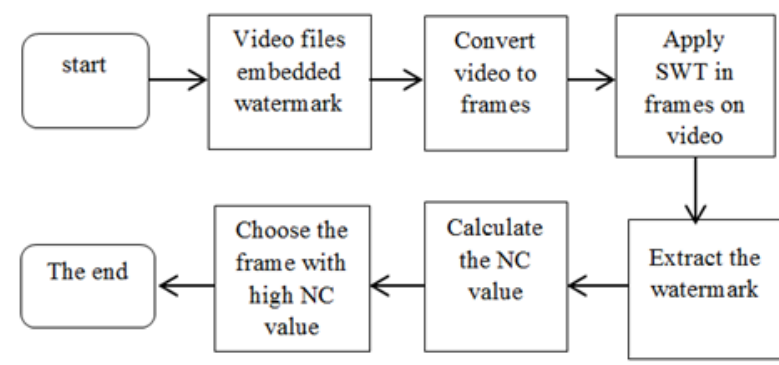

Fig 4: the SWT extracting proposed approach

1. Read the video VI' which embedded the watermark.

2. Convert every frame $f^{\prime}$ of video VI' from RGB to Ycbcr.

3. Apply SWT transform on every frame of video frames VI' and obtained the four beams (horizontal, vertical, diameter and approximations).

4. Extract the watermark values from frames of video VI' depend on the embedding key depend on equation (6):

$\mathrm{Wi}=\left\{\begin{array}{c}0 \cdot(\text { newswa }(\text { Keys })-\mathrm{swa}(\text { Keys })) / \alpha=0 \\ 1 \text { otherwise }\end{array}\right.$

This operation continues until extract the all values of watermark. Where:

Newswa: the matrix of frames which embedded the watermark.

Swa: the matrix of origin frame (the origin cover).

$\alpha$ : the power embedding operand.

Keys: the random keys which we generated in step 5 in embedding operation.

5. Calculate the normalized correlation $\mathrm{NC}$ for every frame of video VI' to measure quality the extracted watermark after that choose the frame with high NC enclosed by the key which we used to embedding operation.

\section{THE GENETIC ALGOARITHM}

Consider the GA on of the important technique in search for the best solution between group of solutions available for specific design, the basic principle of GA depend on the evolution ,algorithm usually start generate group of solution like individuals (chromosomes) difference represent it depend on the chosen problem, this group called (initial population), calculate the evaluation function in every generation for every chromosomes individually, choose the chromosomes randomly or the best of it depend on evaluation function after that apply some special operations (crossover ,mutation, choose) on all individuals of output population for build new population in hope to be best from the previous, repeat the operation of generate the new populations until arrive to a specific condition like determine number of generate the populations or found the demand solution [11].

\section{STEPS EMBEDDING ALGOARITHM APPROACH BY USE GA}

We assume steps of apply GA algorithm on the watermark system by SWT approach for every frame of video frames in 
the next and in fig (5):

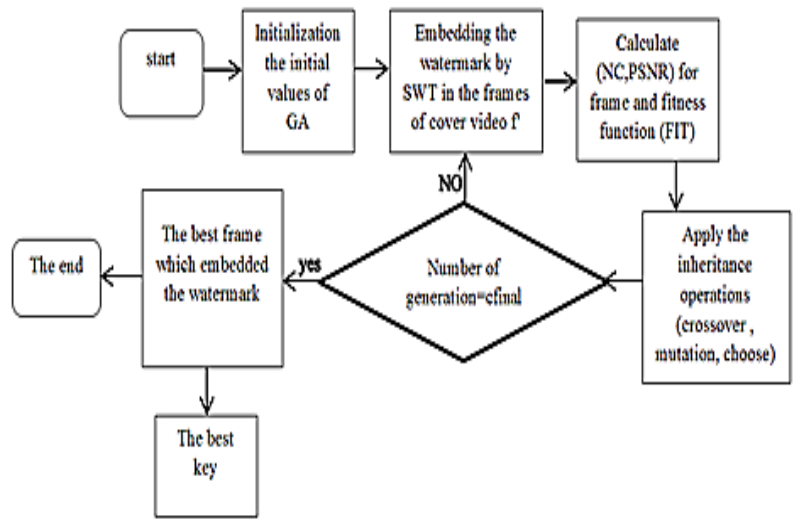

Fig 5: layout of embedding the watermark by use the proposed method SWT \& \&GA

1. Generate $\mathrm{K}$ keys randomly which represent the individual of initial generation.

2. Embedding the watermark W by use SWT approach in every frame of video frames VI by use the key which generate in step 1 one after one, where generate for every frame group of images which the watermark embedded in it and number it $\mathrm{K}$ equal to number of current individual generation for GA.

3. Calculate the PSNR value for every frame for evaluation him quality.

4. Apply attacks on all images which embedded the watermark in it for every frame, after that extract the watermark and calculate the NC for every watermark we extract it.

5. Calculate the fitness function for every individual depend on the PSNR value and sum of all normalized correlation for all extracted watermark from influence of the attacks in the same individual, in as much the value of PSNR bigger than $\mathrm{NC}$ so we enlarge the $\mathrm{NC}$ by multiplex the value of $\mathrm{NC}$ by weight operand $(\lambda)$ for achieve the balance and notice the changes in the values of PSNR and NC which we can notice in equation (7):

Fitness $=P S N R+\lambda * \sum_{i=1}^{n} \operatorname{NCC}\left(W, W^{\prime}\right)$.

Where $\mathrm{n}$ represent the number of attacks which we apply in the this individual. We choose the individuals which have the higher value for fitness function on the current generation which represent the best positions for embedding method.

6. We stop if was the counter of genetic algorithm equal to the final counter cfinal.

7. Execute crossover operation for generate new individual $\mathrm{s}$ for next generation, after that we apply the genetic mutation.

8. Increment the counter of genetic algorithm and back to the step 2 .

Finally, the result of genetic algorithm it the best key for every frame (the individual which has the higher value for fitness function) which we will use it for embedding and extracting the watermark.

\section{STANDARDS EVALUATE THE EFFICENCY}

Calculate error ratio between the origin cover and the cover embedded the watermark by PSNR value in equation (8) [5]:

$$
\text { PSNR }=10 \cdot \log _{10}\left(\frac{\mathrm{MAX}_{\mathrm{I}}^{2}}{\mathrm{MSE}}\right) \mathrm{db}
$$

Where maxi the max value for color density which equal 255 when represent the color ingredient in 8 digits. whereas MSE (Mean Square Error) represent the difference between the symmetry pixels in two images updated and origin in equation (9) $[12]:$

$$
\operatorname{MSE}=\frac{1}{m * n} \sum_{i=1}^{m} \sum_{j=1}^{n}[I(i, j)-\operatorname{Iw}(i, j)]^{2}
$$

Where $I(i, j)$ represent the value of the origin pixel and $\operatorname{Iw}(i, j)$ represent the value of pixel in the cover which contained the watermark, whereas $n, m$ values origin cover dimension. Should the extracted watermark similar to the origin watermark by calculate the match ratio from calculate normalize correlation $\mathrm{NC}$ in equation (10) [12]:

$$
\mathrm{NC}=\frac{\sum_{\mathrm{i}=1}^{\mathrm{m} 1} \sum_{\mathrm{j}=1}^{\mathrm{m} 2}(\mathrm{~W}(\mathrm{i}, \mathrm{j})-\mathrm{mean}(\mathrm{W})) *\left(\mathrm{~W}^{*}(\mathrm{i}, \mathrm{j})-\operatorname{mean}\left(\mathrm{W}^{*}\right)\right)}{\sqrt{\sum_{\mathrm{i}=1}^{\mathrm{m} 1} \Sigma_{\mathrm{j}=1}^{\mathrm{m} 2}(\mathrm{~W}(\mathrm{i}, \mathrm{j})-\mathrm{mean}(\mathrm{W}))^{2} * \sum_{\mathrm{i}=1}^{\mathrm{m} 1} \Sigma_{\mathrm{j}=1}^{\mathrm{m} 2}\left(\mathrm{~W}^{*}(\mathrm{i}, \mathrm{j})-\operatorname{mean}\left(\mathrm{W}^{*}\right)\right)^{2}}}
$$

Where $\mathrm{W}(\mathrm{i}, \mathrm{j})$ represent the value of pixel for origin watermark , $W^{*}(i, j)$ represent the value of extracted pixel for watermark, mean $(\mathrm{w})$ the average value for origin watermark, mean $\left(\mathrm{w}^{*}\right)$ the average value for extracted watermark and $\mathrm{m} 1, \mathrm{~m} 2$ the dimensions of origin watermark.

\section{RESULT AND DISCUSSION}

Applied the proposed method on standard video called foreman from avi from (where avi consist of simple architecture and high quality for image and sound accorder with all operating systems and don't need for additional programs for operating it in [12] the other hand we can use another forms like MP4 but its architecture more complex than avi)with dimension $256 * 256$ with size $8 \mathrm{MB}$ for period time 10 seconds average 30 frame in second and which represent the cover of watermark, the number of frames which consist the video 300 frame. Whereas the used watermark was binary image with size 350byte dimension $32 * 32$. We executed the proposed method by use Matlab.13 with computer properties: processor speed 2.5GHZ size memory $1.98 \mathrm{~GB}$, we applied many attacks to the video for test power and efficient of proposed method in embedding watermark from this attacks salt\&\&pepper with density 0.005 , copying 20 row 20 column randomly, compression from form jpeg with quality factor $\mathrm{Q}=50$ and some filters like Gaussian filter with window $5 * 5$. The results obvious in the table (1) additional the figure (6) the first origin frame (5-a) and the first watermark embedded frame (5-b) for video foreman and the watermark (5-c) which should have embedded. 


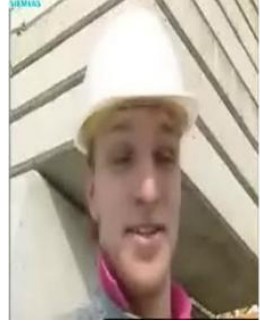

(a-6)

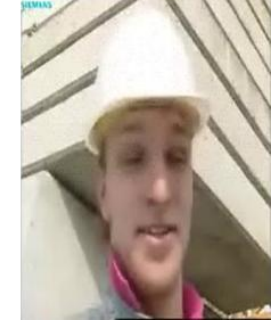

(b-6 )

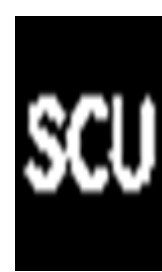

(c-6)
Fig 6 : the first origin frame (a-6) the second embedded frame (b-6) the watermark (c-6).

Table 1. Table compare between the proposed method without and with the GA explicated the values of PSNR, NC

\begin{tabular}{|c|c|c|c|c|c|}
\hline \multirow[t]{2}{*}{ attack } & \multirow[t]{2}{*}{ value } & \multicolumn{2}{|c|}{$\begin{array}{c}\text { The proposed method with } \\
\text { GA }\end{array}$} & \multicolumn{2}{|c|}{$\begin{array}{l}\text { The proposed } \\
\text { method without GA }\end{array}$} \\
\hline & & $\mathrm{NC}$ & PSNR & $\mathrm{NC}$ & PSNR \\
\hline \multirow{2}{*}{ Copying } & 20 & 0.94831 & 42.2349 & 0.9365 & 37.16 \\
\hline & 30 & 0.9041 & 42.2418 & 0.87761 & 37.1742 \\
\hline \multirow{2}{*}{$\begin{array}{c}\text { Gaussian } \\
\text { filter }\end{array}$} & $5 * 5$ & 0.9999 & 42.2582 & 0.99809 & 37.1799 \\
\hline & $7 * 7$ & 0.99981 & 42.2731 & 0.99619 & 37.1843 \\
\hline \multirow{2}{*}{$\begin{array}{c}\text { JPEG } \\
\text { Compres } \\
\text { sion }\end{array}$} & 50 & 0.99066 & 42.2306 & 0.93057 & 37.1827 \\
\hline & 60 & 0.99637 & 42.2303 & 0.95715 & 37.185 \\
\hline \multirow{2}{*}{$\begin{array}{c}\text { Salt and } \\
\text { Pepper } \\
\text { noise }\end{array}$} & 0.005 & 0.98989 & 42.2547 & 0.98954 & 37.2435 \\
\hline & 0.008 & 0.98531 & 42.2514 & 0.9852 & 37.1753 \\
\hline
\end{tabular}

After combine the SWT with GA on the video file we obtained results for PSNR\&\&PC better than SWT without GA and the obvious in table (1) where $\mathrm{NC}=0.99$ at most of attacks and this value near from value 1 that mean the extracted watermark was match or similar to the origin watermark. to measure quality, the proposed method with GA we use the standard PSNR where was its value almost equal $42.27 \mathrm{~dB}$ and this value escape from the visible attack caused by the human eye because this value $42.27 \mathrm{db}$ bigger than $36 \mathrm{db}$. [13] Moreover the proposed method more resist to compression attacks until in use the compression attacks with low quality.

We calculated the overall time complex to two levels embedding and extracting for proposed method with and without GA shown in table (2).

Table 2. Table compare the overall time between the proposed method with and without GA

\begin{tabular}{|c|c|c|}
\hline & $\begin{array}{c}\text { The proposed } \\
\text { method with GA }\end{array}$ & $\begin{array}{c}\text { The proposed } \\
\text { method without } \\
\text { GA }\end{array}$ \\
\hline Time (second) & 700 & 550 \\
\hline
\end{tabular}

In spite of the time complex because using the GA but this increment not too big by percentage $30 \%$ but using the GA caused more enhancing in the quality of frame (PSNR) embedded to the watermark consequently less blurring in the frame, we obtained values for operands NC near from 1 consequently more efficiency in embedding the watermark in the frame and this obvious in table (1). using the GA cause the balance between properties of transparence and effective for proposed system of watermark although the increment of time complex.

) We compare the proposed method by using GA with previous search prepared from Shelke\&\&Chatur [7] where they used transform with GA additional another transforms. We used the same previous standard video foreman with the same number of frames, average of frames, the size, the accuracy, period time and the same previous watermark. We applied many attacks for test robustness and power the embedded watermark in the video, from this attacks noise attack salt\&\&pepper with density 0.005 , filter attack median filter with window $3 * 3$, noise attack Gaussian filter with average $\mu=0$ and contrast $\sigma=0.001$ and ration attack in specific angle which results obvious in table (3).

Table3. Table compare between the proposed method and the previous method [7] with PSNR\&\&NC

\begin{tabular}{|c|c|c|c|c|c|}
\hline \multicolumn{2}{|c|}{} & \multicolumn{2}{c|}{$\begin{array}{c}\text { The previous } \\
\text { method [7] }\end{array}$} & \multicolumn{2}{c|}{$\begin{array}{c}\text { The new proposed } \\
\text { method }\end{array}$} \\
\hline attack & value & NC & PSNR & NC & PSNR \\
\hline $\begin{array}{c}\text { Median } \\
\text { filter }\end{array}$ & $3 * 3$ & 0.9782 & 36.92 & 0.9975 & 38.38 \\
\hline Rotation & 5 & 0.9404 & 33.85 & 0.9753 & 38.65 \\
\hline $\begin{array}{c}\text { Gaussian } \\
\text { noise }\end{array}$ & 0.001 & 0.4986 & 28.74 & 0.8064 & 38.86 \\
\hline $\begin{array}{c}\text { Salt\& } \\
\text { Pepper } \\
\text { noise }\end{array}$ & 0.005 & 0.5510 & 29.32 & 0.9073 & 38.36 \\
\hline
\end{tabular}

Notice from the table (3) that the new proposed method better than the method [7] because obtain on higher value in PSNR\&\&NC, Also notice closeness in the values of PSNR $\& \& N C$ in the two methods expect (salt\&\&pepper, Gaussian) attack the value of PSNR down to $(29.32,28.74)$ in method [7] that because embed data of watermark in LL spam which contains most of signal power so add any noise or blurring cause down in the value of PSNR, also add noise to the LL spam which contain all information of watermark cause changing in it so extract the data of watermark blurrily and this obvious in table (3) where down the value of NC $(0.55,0.49)$ for noise attack whereas in the proposed method distributed the data of watermark in all section of frame on opposite method [7] which use part of frame LL for embedded the watermark in it where notice that the value of NC \&\& PSNR in proposed method better than the method [7] against many attacks.

\section{CONSULION}

consider the new proposed method in this paper from the strongest methods where characterized this method with power , robustness and effective against many attacks which exposed the watermark for change it or delete it, in additional characterized the watermark invisible in the frame which embedded in it and this embed doesn't cause any blurring or change in the holder cover (video), finally we obtained on 
excellent values for PSNR \&\& NC moreover this values gave high quality and efficient for this proposed method in this search.

\section{REFERENCES}

[1] Obimbo C., Salami B., 2012 Using Digital Watermarking for Copyright Protection. In: Gupta M.D., WatermarkingVolume 2, Mithun Das Gupta.

[2] Aslantas V., 2008 A singular-value decomposition-based image watermarking using genetic algorithm, International Journal of Electronics and Communications.

[3] Vikas Sharma and P.S Mann., 2017 A Review on Various Optimized Image Watermarking Techniques. International Journal of Computer Applications., institute of Engineering \& technology, Jalandhar.

[4] Vikas Sharma., P.S Mann., 2017 Watermark Image Enhancement from various Attacks by SWT Using Hybrida Meta-heuristics. International Journal of Engineering and Computer Science.

[5] Mrunalini Madhavrao Chaudhari., 2015 Stationary Wavelet-Transforme Based Steganography for Transmitting Images. International Journal Of Innovative Research In Science Engineering And Technology.

[6] Jumana Waleed ., Thekra Abbas., 2014 A Survey of Digital Image Watermarking Optimization Based on Nature Inspired Algorithme NIAs. International Journal of
Security and Its Applications.

[7] Shelke N.A., Dr. Chatur P.N., 2014 Blind Robust Digital Video Watermarking Scheme Using Hybrid Based Approach. International Journal of Computer Science and Information Technologies.

[8] Sadek R., 2012 SVD Based Image Processing Applications. State of The Art, Contributions and Research Challenges.

[9] Elahian A., Khalili M., Shokouhi S., 2011 Improved Robust DWT-Watermarking in Ycbcr Color Space, Global Journal of Computer Application and Technology.

[10] Rafizul Haque S.M., 2008 Singular Value Decomposition And Discrete Cosine Transform Based Image Watermarking. Scientific Journal of Informatics.

[11] Pan J.S., Huang H.C., Jain L.C.,2004 Intelligent Watermarking Techniques, World Scientific Publishing Co.

[12] Goyal D., Singh R., Hemrajani N.,2014 Comparative Study of Spatial Watermarking of Various Video formats. Journal of Advanced Computing and Communication Technologies.

[13] Roy R., Changder S., Sarkar A., Debnath N.C., 2013 Evaluating Image Steganography Techniques. Future Research Challenges. IEEE International Conference on Computing, Management and Telecommunications. 